\title{
DIMENSÕES QUALITATIVAS E QUANTITATIVAS DO TRABALHO HOSPITALAR: interface com a cultura de segurança do paciente
}

\author{
QUALITATIVE AND QUANTITATIVE \\ DIMENSIONS OF HOSPITAL WORK: \\ interface with the patient safety culture
}

\begin{abstract}
Fabieli Borges', Denise de Fátima Hoffmann Rigo', Thaís Vanessa Bugs', Andressa Morello Kawamoto', João Lucas Campos de Oliveira², Ronaldo Luiz Barboza ${ }^{3}$, Anair Lazzari Nicola ${ }^{4}$
\end{abstract}

\section{RESUMO}

Objetivou-se analisar a cultura de segurança do paciente entre a equipe multiprofissional hospitalar quanto às dimensões que tratam do qualitativo organizacional e o quantitativo de pessoal. Procedeu-se pesquisa descritiva, quantitativa, com a participação de 71 profissionais de um hospital de ensino público do interior do Paraná, que responderam ao questionário "Hospital Survey on Patient Safety Culture". A análise estatística descritiva foi procedida às dimensões de aprendizado organizacional (qualitativa) e dimensão de pessoal (quantitativa) do referido instrumento. A maioria $(67,6 \%)$ dos sujeitos legitimou percepção favorável ao aprendizado organizacional contínuo, contudo, $91,6 \%$ dos profissionais sinalizaram que 0 hospital empregador não propicia quantitativo adequado de pessoal para o atendimento seguro, além das pessoas estarem expostas à elevada carga de trabalho e continuamente atuarem sob tensão. Concluiuse que, através desta dualidade na percepção dos participantes, fica evidente a maior e melhor atenção das lideranças à provisão de capital humano adequado no serviço.

Descritores: Segurança do Paciente; Cultura Organizacional; Downsizing Organizacional; Gestão da Qualidade; Administração Hospitalar.

\section{ABSTRACT}

This study aimed to analyze the patient safety culture among hospital multidisciplinary team as the dimensions dealing with organizational qualitative and quantitative personnel. The procedure was descriptive, quantitative research, with the participation of 71 professionals from a public teaching hospital in the interior of Paraná, who responded to the questionnaire "Hospital Survey on Patient Safety Culture". Descriptive statistical analysis was proceeded to organizational learning dimensions (qualitative) and dimension of staff (quantitative) of the said instrument. Most $(67,6 \%)$ of the subjects legitimized favorable perception to continuous organizational learning, however, $91,6 \%$ of the respondents signaled that the employer hospital provides no quantitative suitable personnel for safe care, in addition to people being exposed to high workload and continuously act under stress. It was concluded that through this duality in the perception of the participants, it is clear the best attention of leaders to the provision of adequate human capital in the service.

Descriptors: Patient Safety; Organizational Culture; Personnel Downsizing; Quality Management; Hospital Administration.
${ }^{1}$ Graduada em Enfermagem pela Universidade Estadual do Oeste do Paraná (UNIOESTE),

Cascavel, PR, Brasil.

${ }^{2}$ Mestre em Enfermagem pela Universidade Estadual de Maringá (UEM), Maringá, PR, Brasil.

${ }^{3}$ Especialista em Filosofia do Direito pela Universidade Estadual do Oeste do Paraná (UNIOESTE), Cascavel, PR, Brasil.

${ }^{4}$ Doutora em Enfermagem Fundamental pela Universidade de São Paulo (USP), São Paulo, SP, Brasil. 


\section{Introdução}

No setor saúde, face especialmente ao avanço científico-tecnológico acelerado; a necessidade de coadunar propósitos organizacionais com a satisfação de consumidores; bem como o dever de os serviços promoverem a redução dos riscos associados à assistência, hodiernamente, há uma preocupação de gestores de saúde crescente no que tange à busca pela qualidade no cuidado.

Ante ao exposto, tem-se que, sob influência de conhecimentos essencialmente administrativos, na área da saúde, a qualidade é um bem desejável que deve estar em consonância com a prática profissional; e, para isso, é indispensável que a qualidade seja gerida sistêmica e sistematicamente, a fim de promover mudanças e o (re)planejamento cíclico com vistas, inclusive, à satisfação e a segurança dos clientes ${ }^{1}$.

Corroborando com a ideia explicitada, no contexto brasileiro, a Agência Nacional de Vigilância Sanitária (ANVISA) e o Ministério da Saúde vêm desenvolvendo ações visando ampliar a difusão dos conhecimentos e práticas que regem a segurança no atendimento à saúde, que é um dos componentes críticos da qualidade ${ }^{2}$. Destarte, o Programa Nacional de Segurança do Paciente (PNSP) foi instituído por meio da Portaria $n^{\circ} 529$ de $1^{\circ}$ de Abril de 2013, com o objetivo geral de contribuir para a qualificação do cuidado em saúde em todos os estabelecimentos de saúde do território nacional ${ }^{3}$.

Sob o entendimento que a qualidade do cuidado em saúde é indissociável à segurança no mesmo, entende-se a segunda como a "redução a um mínimo aceitável do risco de dano desnecessário ao cuidado de saúde"2. Diante disso, é notório que a segurança do paciente deve ser vista como uma meta incessante de busca pelas organizações de saúde, contudo, devido às próprias características do labor deste peculiar setor de produção, o atendimento seguro é um bem complexo e dinâmico, que muito dificilmente será almejado com eficiência e eficácia caso não haja real compromisso coletivo frente ao mesmo ${ }^{4}$.

Com base no que foi explanado, alude-se que um dos pilares que sustenta a segurança do paciente nas instituições de saúde é a cultura organizacional das mesmas ${ }^{2-5}$. Para tanto, esta "cultura de segurança" deve ser composta, entre outros, por linhas de comunicação efetivas; confiança mútua entre os membros da equipe multiprofissional; aprendizado organizacional contínuo; compromisso da alta gestão com a segurança; liderança; abordagem não punitiva ao erro, entre outros ${ }^{6}$.

Fica evidente que a cultura de segurança do paciente nada mais é do que uma postura organizacional sistêmica frente a este bem desejável2; e, neste aspecto, é esperado que a cultura, já que esta não faz parte do aparato formal e visível da instituição de saúde, seja multifacetada e complexa ${ }^{7}$. No contexto da segurança isso não é diferente, posto que são diversos os aspectos que podem influenciar positiva ou negativamente a cultura, como alguns já supracitados, ao exemplo do aprendizado organizacional contínuo e também, outro aspecto relacionado ao capital humano das organizações, que é o quantitativo de pessoal ${ }^{8}$.

Talvez elencar o avanço do qualitativo organizacional ao aprendizado contínuo, bem como o quantitativo de pessoal como elementos que interferem na segurança do paciente e, por consequência, na cultura de segurança da organização, seja uma obviedade, dado que já é amplamente conhecido que o trabalho em saúde é uma ação essencialmente humana, em que a produção e o consumo do "produto" (a assistência) acontecem sinergicamente durante a relação de pessoas ${ }^{9}$. Apesar disso, também não é novidade, tampouco de domínio exclusivo da comunidade científica, que o setor saúde brasileiro vivencia diariamente dificuldades relacionadas ao déficit dos mais diversos recursos elou possibilidades, o que não raramente inclui a deficiência do aprendizado organizacional contínuo e também, do quantitativo adequado de pessoal.

O panorama antes referido possivelmente é intensificado no contexto hospitalar, onde a densidade tecnológica no atendimento tende a ser aumentada, além da maior complexidade assistencial, o que resulta numa comum atribulação do processo de trabalho, favorecendo dificuldades (intrínsecas ao serviço) mais evidentes de proporcionar o atendimento seguro ${ }^{4-5-6}$.

Cabe aludir, ante ao exposto, que investigar sobre a interface do aprendizado organizacional contínuo e a adequação de pessoal, partindo da premissa de que isso pode significar dimensões qualitativas e quantitativas da força de trabalho hospitalar na interface com a segurança do paciente, certamente é importante porque, pode fundamentar o (re)direcionamento de ações gerenciais e a tomada de decisão que sejam convergentes ao avanço da segurança no atendimento. Reforça essa justificativa o fato de que, ainda que as temáticas segurança e cultura de segurança do paciente pareçam ser expoentes na produção científica, há recomendação nacional recente ${ }^{10}$ de que há necessidade eminente de ampliar o diagnóstico das questões que envolvem a segurança do paciente no Brasil, o que sem dúvida inclui as dimensões antes referidas.

Com base no enunciado anterior, questiona-se: Qual é a percepção de profissionais hospitalares em relação ao aprendizado contínuo e à adequação de pessoal, na sua relação com a segurança do paciente? $\mathrm{E}$, para responder a este questionamento, o objetivo deste estudo consiste em analisar a cultura de segurança do paciente entre a equipe multiprofissional hospitalar quanto às dimensões que tratam do qualitativo organizacional e o quantitativo de pessoal. 


\section{Metodologia}

Trata-se de um estudo descritivo, transversal e quantitativo. Foi desenvolvido em um hospital universitário público, situado em uma região polo do interior do Paraná, que tem capacidade operacional de 195 leitos, todos conveniados ao Sistema Único de Saúde (SUS). Esta instituição hospitalar destina-se principalmente para o atendimento de alta complexidade, para uma população de aproximadamente dois milhões de habitantes.

A saber, os locais de investigação foram as unidades de Clínica Médica e Cirúrgica Geral (F2); Ortopedia e Neurologia (G3); e Centro Cirúrgico (CC) do referido hospital. As unidades foram escolhidas intencionalmente, bem como, contam respectivamente com 28 e 26 leitos, e cinco salas operatórias.

A população em potencial a ser investigada consistiu todos os profissionais (171) que atuavam diariamente nos campos estudados no período de abril a junho de 2014, quais sejam: enfermeiros, técnicos e auxiliares de enfermagem, médicos do corpo clínico, farmacêuticos, fisioterapeutas; e residentes de medicina, enfermagem, farmácia e fisioterapia.

A amostra estudada foi conformada em consonância aos seguintes critérios de elegibilidade: foi considerado critério de exclusão para os profissionais de enfermagem (únicos com escala de trabalho prevista): férias, licenças de qualquer natureza ou outros motivos. Considerou-se recusa à participação, aqueles profissionais (qualquer categoria) que se resignaram em preencher o instrumento de coleta de dados após três tentativas. Como os demais profissionais previstos à participação não possuíam escala de trabalho fixada, se estabeleceu como critério de inclusão dos mesmos estarem presentes nas unidades durante o período de coleta de dados.

Foram considerados, ainda, outros procedimentos de refinamento (exclusão) para a definição da amostra investigada. Estes, referentes ao instrumento de coleta de dados utilizado, tais como: os que não continham pelo menos uma sessão respondida por completo; que continham menos que a metade dos itens preenchidos; e/ou que continham a mesma resposta em todos os itens.

Com base nos critérios de elegibilidade, a amostra analisada foi composta por 71 profissionais da equipe multiprofissional que responderam ao questionário "Hospital Survey on Patient Safety Culture" (HSOPSC), validado e adaptado à cultura brasileira ${ }^{11}$. Ademais, os participantes também responderam questões referentes à sua caracterização sociodemográfica e laboral.

A abordagem de todos os participantes (e os potenciais) se deu no próprio local de trabalho durante o início dos turnos nas unidades investigadas, no decorrer do período de coleta de dados. Todos os profissionais foram informados sobre 0 objetivo do estudo e a voluntariedade na participação, bem explicitação da necessidade de leitura e assinatura do Termo de Consentimento Livre e Esclarecido (TCLE) fornecido, igualmente assinado em duas vias de igual teor pelo pesquisador, e preenchimento do questionário. Ao final dos turnos, o pesquisador retornava aos campos de investigação para recolhimento dos questionários.

O instrumento utilizado contém 42 questões relacionadas à cultura de segurança do paciente, as quais são agrupadas em 12 dimensões ${ }^{11-8}$. Visando o pleno atendimento do objetivo desta investigação, optou-se por analisar as dimensões "Aprendizado organizacional - melhoria contínua"; e "Pessoal", partindo da prerrogativa de que essas dimensões melhor refletem as dimensões qualitativas e quantitativas da força de trabalho hospitalar, na sua interface com a cultura de segurança dos profissionais - objetos de estudo.

O HSOPSC inclui itens que são avaliados a partir de uma escala de Likert de cinco pontos, com categorias de respostas em grau de concordância. A avaliação de cada dimensão e item é estimada com base no percentual de respostas. Valores percentuais mais altos ou mais baixos indicam percepções positivas/negativas em relação à cultura de segurança do paciente, dependendo da assertiva que foi assinalada no item de avaliação $0^{11-8}$. O grau de concordância das dimensões analisadas está disposto às notações "DF" - discordo fortemente; e "D" - discordo; "N" - neutro; "C" - concordo; e "CF" - concordo fortemente.

Os dados coletados à aplicação do instrumento e às questões de caracterização foram tabulados em planilhas eletrônicas do Microsoft Office Excel 2010. Após isso, procedeu-se a análise estatística descritiva dos dados, com o uso da mesma ferramenta tecnológica. As variáveis sociodemográficas e laborais foram utilizadas para traçar o perfil da amostra, e as variáveis concernentes às dimensões da cultura de segurança, para analisar os aspectos relacionados ao aprendizado contínuo organizacional e quantitativo de pessoal, na sua interface com a cultura de segurança do paciente.

Cumpre salientar que todas as exigências éticas previstas na Resolução 466/2012, que regem as pesquisas envolvendo seres humanos, foram integralmente respeitadas. Ademais, o Projeto que fomentou este estudo foi submetido e aprovado (protocolo 558.430/2014) pelo Comitê de Ética em Pesquisa da Universidade Estadual do Oeste do Paraná. 


\section{Resultados e Discussão}

Conforme exposto, após aplicação dos critérios de elegibilidade, esta investigação contou com a participação de 71 profissionais hospitalares da equipe multiprofissional. Quanto aos dados sociodemográficos, constatou-se que a maioria (66,2\%) era do sexo feminino; possuíam de 31 a 40 anos (32,4\%); seguido de 20 a 30 anos (23,9\%); e apenas um participante $(1,4 \%)$ tinha mais de 51 anos. A equipe de enfermagem, incluindo os residentes desta categoria, foi predominante $(64,8 \%)$ na amostra. Quanto aos demais profissionais, houve a participação de sete médicos do corpo clínico (9,9\%); um farmacêutico (1,4\%); dois fisioterapeutas (2,8\%), além de quatro residentes de fisioterapia (5,6\%); um residente de farmácia $(1,4 \%)$; e 10 residentes de medicina $(14,1 \%)$.

Fica evidente que os resultados descritos reafirmam que a equipe de enfermagem tem a maior representatividade no quantitativo do capital humano hospitalar. Ademais, é possível aludir também que a maior participação de mulheres na amostra pode estar atrelada à predominância da enfermagem na investigação, visto que esta categoria tem o cuidado como finalidade primária e essência da profissão, que historicamente remonta-se à figura da mulher, esta vista como provedora do cuidado familiar.

Conforme já explanado, a cultura de segurança do paciente, à sua essência positiva, deve ser um bem comum entre indivíduos e organização, com vistas ao favorecimento do atendimento seguro ao cliente ou usuário de saúde ${ }^{2}$, que, afinal, é o consumidor do processo de trabalho neste setor de produção. Em vista do caráter sistêmico que a cultura de segurança carrega à sua existência, é importante e necessário que o aprendizado contínuo, buscando melhorias cíclicas, seja fomentado na organização ${ }^{4}$. Neste aspecto, a Tabela 1 sumariza os resultados obtidos à análise da dimensão de cultura de segurança do paciente "Aprendizado organizacional - melhoria contínua".

Tabela 1 - Distribuição das frequências das respostas da dimensão de cultura de segurança do paciente: "aprendizado organizacional - melhoria contínua". Cascavel (PR), 2014.

\begin{tabular}{|c|c|c|c|c|c|c|c|}
\hline Item & & (DF) & (D) & (ND) & (C) & (CF) & Total \\
\hline $\begin{array}{l}\text { (B6) Os profissionais estão } \\
\text { continuamente desenvolvendo ações } \\
\text { para melhorar a segurança do paciente }\end{array}$ & $\begin{array}{l}\mathrm{N} \\
\%\end{array}$ & 0 & $\begin{array}{l}15 \\
21,1\end{array}$ & 8 & $\begin{array}{c}42 \\
59,2\end{array}$ & $\begin{array}{l}6 \\
8,4\end{array}$ & $\begin{array}{c}71 \\
100,0\end{array}$ \\
\hline $\begin{array}{l}\text { (B9) Erros têm levado a } \\
\text { mudanças positivas }\end{array}$ & $\begin{array}{l}\mathrm{N} \\
\%\end{array}$ & $\begin{array}{r}2 \\
2,9 \\
\end{array}$ & $\begin{array}{r}18 \\
25,7 \\
\end{array}$ & $\begin{array}{r}15 \\
21,4 \\
\end{array}$ & $\begin{array}{r}33 \\
47,1 \\
\end{array}$ & $\begin{array}{r}2 \\
2,9 \\
\end{array}$ & $\begin{array}{r}70^{*} \\
100,0\end{array}$ \\
\hline $\begin{array}{c}\text { (B13) Depois que mudanças para } \\
\text { melhorar a segurança do paciente são } \\
\text { implantadas, a sua efetividade é avaliada. }\end{array}$ & N & $\begin{array}{l}1 \\
1,4\end{array}$ & $\begin{array}{l}29 \\
40,9\end{array}$ & $\begin{array}{l}14 \\
19,7\end{array}$ & $\begin{array}{l}26 \\
36,6\end{array}$ & $\begin{array}{l}1 \\
1,4\end{array}$ & $\begin{array}{c}71 \\
100,0\end{array}$ \\
\hline
\end{tabular}

*Itens em branco não foram considerados.

De maneira geral, é possível perceber (Tabela 1) que os resultados analisados à dimensão de aprendizado organizacional contínuo, neste estudo compreendido como uma perspectiva qualitativa do trabalho em saúde refletiu, na maioria, percepções positivas dos profissionais participantes. Ao exemplo disso, 67,6\% dos entrevistados sinalizaram que existe o desenvolvimento contínuo de ações para a melhoria da segurança do paciente na instituição hospitalar empregadora.

$O$ desenvolvimento de ações com vistas ao atendimento seguro como produto do trabalho em saúde vai encontro dos princípios que regem a gestão pela qualidade, especialmente quando a comunicação (produto de uma ação planejada) entre setores é eficaz, e a disseminação de estratégias exitosas se estende sistemicamente ${ }^{12}$. Neste sentido, os profissionais, ao concordarem em sua maioria que existe o (re)planejamento estratégico de ações cíclicas de melhorias, legitimam um aspecto da cultura de segurança do paciente, no âmbito da dimensão do aprendizado organizacional.

Cabe reafimar que, entende-se aqui que a dimensão do aprendizado organizacional, este, podendo ser promotor de melhorias contínuas, pode ser traduzida como uma dimensão qualitativa do trabalho hospitalar porque, sabidamente, o trabalho em saúde permeia a prestação de serviços peculiares e complexos, que podem ser influenciados, à sua qualidade, pela forma (ou a valorização) que a organização proporciona e avalia ações de aprendizado, tanto no âmbito organizacional, como aos profissionais ${ }^{4-13}$.

Ante ao exposto, cumpre mencionar que a organização preocupada em desenvolver-se, bem como desenvolver seu capital humano, pode se utilizar de estratégias variadas que favoreçam o aprendizado contínuo. Na área da saúde, 
uma forma de a instituição aprender questões relacionadas à segurança do paciente, é, justamente, através dos erros². Neste aspecto, outro item avaliado positivamente à percepção dos profissionais frente à dimensão de cultura de segurança que tratou do aprendizado organizacional, apesar da menor expressividade, diz respeito às mudanças positivas após as ocorrências de erros, a qual abarcou a concordância de $50 \%$ dos profissionais à assertiva (B9).

Conceitualmente, no cotidiano laboral, os erros são comumente confundidos com os eventos adversos. Isso porque, os erros consistem em falhas na execução de uma ação planejada que pode resultar um evento adverso², os quais são definidos como incidentes que resultam em danos à saúde do usuário ${ }^{3}$. Destarte, os eventos adversos representam na atualidade um dos maiores desafios para o aprimoramento da qualidade e segurança no setor saúde ${ }^{12}$.

Ante ao exposto, converge-se à reflexão de que o aprendizado organizacional a partir dos erros certamente é uma atividade salutar da instituição de saúde que preza o atendimento seguro porque, através da notificação, análise crítica e medidas de prevenção coletivas do erro, talvez, os eventos adversos poderão ser evitados/minimizados.

Apesar do que foi descrito e debatido, há de se convir que as respostas inerentes ao aprendizado pelo erro não foram tão expressivamente positivas, já que a somatória da neutralidade e das respostas negativas (50\%) se equivale às respostas interpretadas como positivas, o que possivelmente pode significar que esta prática ainda não é totalmente disseminada na organização como um todo.

Em outro item de avaliação (Tabela 1), os profissionais denotaram que, apesar de legitimaram a prática de ações desenvolvidas continuamente para a melhoria da segurança do paciente (B6), estas ações, de acordo com a maioria dos participantes, não são avaliadas (B13). Isso é relevante e preocupante, pois, classicamente, a avaliação é, talvez, a principal ferramenta de gestão que alicerça a qualidade nos serviços de saúde, e por consequência, a segurança, visto que os resultados fornecidos pela avaliação subsidiam a tomada de decisão racional, que irá interferir na busca da melhoria contínua ${ }^{12-14}$.

Em contraste aos resultados descritos, outra pesquisa ${ }^{15}$ que também utilizou o HSOPSC contraria a realidade constatada, ao passo que, também no item que relaciona-se à avaliação posterior às medidas de segurança implantadas, a maior parte da amostra participante neste estudo (45,8\%) discordou da assertiva.

Com base nos resultados descritos e na literatura consultada, percebe-se que a avaliação de medidas de segurança institucionais implantadas, enquanto prática essencialmente gerencial, é um fator que notoriamente é influenciado pelo modelo e a maturidade da gestão organizacional e à sua singularidade; por isso, é esperado que este seja um item de discrepância entre diferentes realidades.

Nos hospitais, como reflexo desta pesquisa, devido à complexidade do serviço prestado, da dinamicidade do processo de trabalho e da possível alta densidade tecnológica, as gerências institucional e departamental se deparam cotidianamente com problemas de difícil resolução, bem como manejo de recursos variados, que devem ser otimizados à sua maior eficiência e eficácia, com vistas à qualidade ${ }^{14}$.

Apesar da variedade de recursos gerenciados continuamente no âmbito hospitalar, este, por se tratar de uma instituição prestadora de serviços de saúde, existe acentuada influência do capital humano. Para tanto, conformando outro relevante aspecto da cultura de segurança do paciente, os resultados da dimensão "Pessoal" estão ilustrados na Tabela 2.

Tabela 2 - Distribuição das frequências das respostas da dimensão de cultura de segurança do paciente:

“Pessoal". Cascavel (PR), 2014.

\begin{tabular}{c|c|c|c|c|c|c|c}
\hline Item & & (DF) & (D) & (ND) & (C) & (CF) & Total \\
\hline $\begin{array}{c}\text { (B2) O quadro de pessoal é suficiente para } \\
\text { desenvolver as atividades }\end{array}$ & $\mathrm{N}$ & 44 & 21 & 1 & 3 & 2 & 71 \\
\hline $\begin{array}{c}\text { (B5) Os profissionais trabalham mais do que } \\
\text { seria desejável para o cuidado do paciente }\end{array}$ & $\mathrm{N}$ & 3 & 15 & 3 & 27 & 22 & $70^{*}$ \\
\hline $\begin{array}{c}\text { (B7) Há necessidade de mais profissionais } \\
\text { temporários/terceirizados do que seria } \\
\text { desejável para o cuidado do paciente }\end{array}$ & $\mathrm{N}$ & 2 & 4 & 3 & 31 & 28 & $68^{*}$ \\
\hline $\begin{array}{c}\text { (B14) Os profissionais trabalham sob tensão, } \\
\text { realizando muitas atividades de forma rápida }\end{array}$ & $\mathrm{N}$ & 0 & 4 & 8 & 37 & 21 & $70^{*}$ \\
\hline
\end{tabular}

*Itens em branco não foram considerados. 
Pode-se notar por meio dos dados apresentados (Tabela 2) que as respostas dos profissionais de saúde, quando questionados no que se refere ao quantitativo de pessoal ser suficiente para prestar assistência pautada na segurança do paciente reflete notavelmente uma percepção negativa dos mesmos.

Não menos importante, nota-se que a expressiva maioria (91,6\%) discordou da assertiva que dizia que o quadro de pessoal é suficiente para desenvolver as atividades, o que pode ser traduzido, à percepção desses sujeitos, como subdimensionamento ou déficit quantitativo de pessoal. $O$ dimensionamento de pessoal, na área da saúde, vem se consolidando como uma ação gerencial realizada e investigada essencialmente na enfermagem, e consiste em ferramenta de planejamento da assistência de modo a equilibrar o quadro de funcionários às necessidades dos usuários, do serviço e da instituição ${ }^{16}$.

Em se tratando da equipe de enfermagem, é importante destacar que esta categoria contempla o maior contingente de profissionais no âmbito hospitalar, e que está em contato ininterrupto com os usuários, exercendo influência direta na segurança do paciente ${ }^{16}$. Neste aspecto, à luz dos resultados descritos, evidencia-se que os profissionais sentemse insatisfeitos com o quantitativo disponível ao trabalho na realidade investigada, o que resultou numa percepção que diverge dos preceitos do atendimento seguro.

O fato constatado neste estudo ganha ainda mais relevância ao passo que, na mesma instituição investigada, existem outras evidências científicas recentes que demonstraram que o número de profissionais de enfermagem é, deveras, insuficiente para atender seguramente a demanda de cuidado ${ }^{17-18}$. Essa realidade se intensifica no contexto da categoria de enfermeiros, que devem ser predominantes no cuidado à pacientes "complexos", ou seja, que demandam cuidados semi-intensivos e intensivos, ocorrência observada em unidades não destinadas para esse tipo de atendimento na instituição ${ }^{18}$, e que também fizeram parte dos locais de investigação (F2 e G3) no presente estudo.

Para calcular o quadro de pessoal adequado (dimensionado) é necessário conhecer a carga de trabalho profissional ${ }^{18}$. Neste aspecto, estudo realizado em outro hospital universitário do sul do Brasil relacionou a carga de trabalho da equipe de enfermagem com o nível de segurança dos pacientes e concluiu que o aumento do número de pacientes por enfermeiros ou técnicos/auxiliares em enfermagem está diretamente relacionado com aumento das reações adversas nos usuários como quedas do leito e infecções no cateter venoso central19.

No que diz respeito à quantidade de trabalho desenvolvida pelos profissionais, os entrevistados, $70 \%$ da amostra concordou com a afirmação de que o trabalho realizado é maior do que seria desejável para o cuidado do paciente. Para tanto, também no contexto da enfermagem, alvitra-se que é necessário utilizar o sistema de classificação de pacientes (SCP) como ferramenta de gestão de recursos humanos no tocante à carga de trabalho, visto que, a enfermagem corresponde à grande maioria dos profissionais nas instituições hospitalares. Destarte, o SCP possibilita calcular quantas horas de trabalho não destinadas aos pacientes, bem como, os recursos humanos específicos de cada setor, favorecendo a segurança dos pacientes ${ }^{18}$.

Cabe aludir, ante ao exposto, que a utilização de ferramentas gerenciais como o SCP e o dimensionamento de pessoal possivelmente devem ser recomendadas por todas as áreas de prestação do cuidado hospitalar. Contudo, 0 consumo acrítico desses conhecimentos muito provavelmente se tornará como um aporte burocrático na instituição, e não um instrumento de gestão para negociação com gestores hospitalares; e, neste sentido, recomenda-se que o uso desses instrumentos de gestão sejam racionalizados e sistematizados no contexto laboral de cada realidade.

Referente ao item B14 que tratou da tensão no trabalho e sobrecarga de atividades contou com mais de $80 \%$ de concordância, o que pode estar intimamente relacionado com o déficit de profissionais e carga de trabalho excessiva. No Brasil, este é um cenário comum, onde a complexidade do Sistema Único de Saúde (SUS), financiado com recursos públicos, acaba se agravando por questões estruturais, políticas, econômicas e culturais ${ }^{19}$.

Ante ao exposto, percebe-se que a percepção dos profissionais participantes em relação às dimensões da cultura de segurança do paciente estudadas se postou como uma dualidade, já que, apesar do aprimoramento organizacional contínuo ter sido legitimado favoravelmente pelos sujeitos, os mesmos sinalizaram que a instituição não provém o quantitativo de recursos humanos (que também trabalha sob tensão) necessário para o atendimento seguro.

\section{Considerações Finais}

Através deste estudo, foi possível analisar duas importantes dimensões que refletem a cultura de segurança do paciente, quais sejam: o aprendizado organizacional contínuo e a dimensão de pessoal, ou capital humano. Nesta investigação, as referidas dimensões foram tratadas como um reflexo qualitativo e quantitativo, respectivamente, no âmbito hospitalar. 
Conclui-se que a percepção dos profissionais do local investigado revelou uma perspectiva dual em relação à segurança do paciente, pois, apesar do aprendizado ou melhoria contínua no âmbito organizacional ser um fator globalmente percebido como favorável, os sujeitos legitimaram que o hospital não propicia quantidade de profissionais suficiente para 0 atendimento seguro, além de o capital humano, por possível consequência, ser exposto à elevada carga de trabalho, bem como atuar sob constante tensão.

Cabe mencionar que este estudo possui limitações como, por exemplo, a ausência de análise estatística inferencial e a pontualidade geográfica, o que impede que os achados sejam inferidos para outras realidades. Apesar disso, acreditase que a pesquisa traz contribuições sólidas ao construto do conhecimento na segurança do paciente, especialmente por evidenciar às lideranças a importância do suprimento adequado de capital humano hospitalar.

Ante ao exposto, alvitra-se que novas investigações são necessárias, com diferentes abordagens metodológicas, ao exemplo dos estudos analíticos com a finalidade de melhor elucidar a relação do quantitativo de pessoal com indicadores de qualidade e segurança do paciente, a fim de sustentar melhor a possível necessidade de contratação de pessoal.

Por fim, vale salientar que 0 atendimento seguro deve ser um fator de atenção e vigília constante em termos institucionais sistêmicos, o que impera que a cultura organizacional seja um elemento, ainda que subjetivo, que sustente outras ações visando às melhorias contínuas.

\section{Referências}

1. Menezes PIFB, D'Innocenzo M. Dificuldades vivenciadas pelo enfermeiro na utilização de indicadores de processos. Revista Brasileira de Enfermagem[Internet]. 2013[citado 24 jul 2015];66(4): 571-577. Disponível em:http://www.scielo.br/ pdf/reben/v66n4/v66n4a16.pdf.

2.Brasil. Ministério da Saúde. Documento de referência para o Programa Nacional de Segurança do Paciente/ Ministério da Saúde; Fundação Oswaldo Cruz; Agência Nacional de Vigilância Sanitária. [Internet] - Brasília(DF):2014.[citado 8 abr 2015]. Disponível em:http://bvsms.saude.gov.br/bvs/publicacoes/documento_referencia _programa_nacional_seguranca.pdf.

3. Brasila. Ministério da Saúde. Portaria No 529 , de $1^{0}$ de abril de 2013. Institui o Programa Nacional de Segurança do Paciente (PNSP). Brasília (DF): 2013. [citado 7 abr 2015]. Disponível em:http://www.cvs.saude.sp.gov.br/up/U_PT-MSGM-529_010413.pdf.

4. Mello JF de, Barbosa SFF. Patient safety culture in intensive care: nursing contributions. TextContextNursing [Internet].2013 [citado 07 maio 2015]; 22(4): 1124-33. Disponível em: http://www.scielo.br/pdf/tce/v22n4/en_31.pdf.

5. Rigobello MCG, Carvalho REFL de, Cassiani SHB, Galon T, Capucho HC, Deus NN de. The climate of patient safety: perception of nursing professionals. Acta Paul Enferm[Internet]. 2012 [citado 24 jun 2015]; 25(5):728-35. Disponível em: http://www.scielo.br/pdf/ape/v25n5/en_13.pdf.

6. Tomazoni A, Rocha PK, Souza S, Anders JC, Malfussi HFC.Cultura de segurança do paciente em unidades de terapia intensiva neonatal.Revista Latino-Americana de Enfermagem [Internet]. 2014 [citado 12 maio 2015]; 22 (5): 755-63. Disponível em:http://www.scielo.br/pdf/rlae/v22n5/es_0104-1169-rlae-22-05-00755.pdf.

7. Kurcgant $P$, Massarollo MCKB. Cultura e poder nas organizações de saúde. In: Kurcgant $P$ (coord). Gerenciamento em enfermagem. 2a ed. Rio de Janeiro: Guanabara Koogan; 2010.p. 23-33.

8. Clinco SDO. O hospital é seguro? Percepções de profissionais de saúde sobre segurança do paciente. [dissertação]. São Paulo: Escola de Administração de Empresas. Fundação Getúlio Vargas, 2007. 98 p. [citado 26 maio 2015]. Disponível em: http://bibliotecadigital.fgv.br/dspace/bitstream/handle/10438/4900/106674.pdf?sequence=1.

9.Souza SS, Costa R, Shiroma LMB, Maliska ICA, Amadigi FR, Pires DEP et al. Reflexões de profissionais de saúde acerca do seu processo de trabalho. Rev. Eletr. Enf. [Internet]. 2010;[citado 03 ago. 2014];12(3):449-55. Disponível em:https://www.fen.ufg.br/fen_revista/v12/n3/v12n3a05.htm.

10. Reis CT, Martins M, Laguardia J. A segurança do paciente como dimensão da qualidade do cuidado de saúde - um olhar sobre a literatura. Ciência \& Saúde Coletiva. [Internet]. 2013 [citado 02 ago. 2015]; 18(7):2029-2036. Disponível em: http://www.scielo.br/pdf/csc/v18n7/18.pdf.

11.Reis CT, Laguardia J, Martins M. Adaptação transcultural da versão brasileira do Hospital SurveyonPatientSafetyCulture: etapa inicial. Cad. Saúde Pública [Internet]. 2012 [citado 10 abr. 2015]; 28(11):2199-2210. Disponível em: http://www. scielo.br/pdf/csp/v28n11/19.pdf. 
12. Silva - Batalha EMS, Melleiro MM. Patient safety culture in a teaching hospital: differences in perception existing in the different scenarios of this institution. TextoContextoEnferm [Internet]. 2015 [citado 23 jun 2015];24(2):432-41. Disponível em: http://www.scielo.br/pdf/tce/v24n2/0104-0707-tce-24-02-00432.pdf.

13. Costa DB, Garcia SD, Vannuchi MTO, Haddad MCL. Impact of staff training in the health working process: an integrative review. J Nurs UFPE [Internet] 2015. [citado 30 jul 2015]; 9 (4): 7439-47. Disponível em: http://www.revista. ufpe.br/revistaenfermagem/index.php/revista/article/view/6555/pdf_7575.

14. PenaMM, Silva SEM, Tronchin DMR, Melleiro MM. The use of the quality model of Parasuraman, Zeithaml and Berry in health services.RevEscEnferm USP [Internet]. 2013 [citado 21 maio 2015]; 47(5):1227-32. Disponível em: http://www. scielo.br/pdf/reeusp/v47n5/0080-6234-reeusp-47-05-1227.pdf.

15. Batalha E.M.S.S. A cultura de segurança do paciente na percepção de profissionais de enfermagem de um hospital universitário.[dissertação]. São Paulo: Escola de Enfermagem da Universidade de São Paulo, 2012.

16. Versa GLGS,Inoue KC, Nicola AL, Matsuda LM. Influência do dimensionamento da equipe de enfermagem na qualidade do cuidado ao pacientecrítico. Texto Contexto Enferm [Internet]. 2011 out-dez [citado 01 jul. 2015]; 20(4): 796802.Disponível em: http://www.scielo.br/scielo.php?pid=S010407072011000400020\&script=sci_arttext.

17. Casarolli ACG, Eberhardt TD, Nicola AL, Fernandes LM. Nível de complexidade assistencial e dimensionamento de enfermagem no pronto-socorro de um Hospital Público. RevEnferm UFSM [Internet]. 2015 [citado 10 ago. 2015]; 5 (2): 278-285. Disponível em: http://cascavel.ufsm.br/revistas/ojs-2.2.2/index.php/reufsm/article/view/16811/pdf.

18. Barbosa HB, PaianoLAG, Nicola AL,Fernandes LM. Nível de complexidade assistencial de pacientes e o quantitativo de profissionais de enfermagem.RevEnferm UFSM[Internet].2014 [citado 10 ago. 2015];4(1):29-37. Disponível em: http:// cascavel.ufsm.br/revistas/ojs-2.2.2/index.php/reufsm/article/view/9230.

19. Magalhães AMM, Dall'Agnol, CM, Marck, PB. Carga de trabalho da equipe de enfermagem e segurança do paciente - estudo com método misto na abordagem ecológica restaurativa. Rev. Latino-Am. Enfermagem [Internet]. 2013 [citado 17 jun. 2015]; 21: 146-154.Disponível em: http://www.scielo.br/scielo.php?pid=S0104-11692013000700019\&script=sci_ arttext\&tlng=pt.

\section{Fabieli Borges}

Endereço para correspondência - Rua: Odontologia, n 834, apto 06, Bairro: Universitário, CEP: 85819-220, Cascavel, PR, Brasil.

E-mail: fabieliborges@yahoo.com.br

Lattes: http://lattes.cnpq.br/5857244387101386

Denise de Fátima Hoffmann Rigo - denisehoffmannrigo@yahoo.com.br

Thaís Vanessa Bugs - theesbugs@hotmail.com

Andressa Morello Kawamoto - morellok@hotmail.com

João Lucas Campos de Oliveira - joao-lucascampos@hotmail.com

Ronaldo Luiz Barboza - ronaldoluizbarboza@gmail.com

Anair Lazzari Nicola - anairln@yahoo.com.br

\section{Enviado em 02 de setembro de 2015. Aceito em 03 de abril de 2016.}

